\title{
The use of colour Doppler imaging in the diagnosis of retinal detachment
}

M Ido, S Osawa, M Fukukita, M Sugimoto, Y Wakitani, Y Ito, M Miyamura, M Sasoh and Y Uji

\begin{abstract}
Design Prospective study.

Purpose To evaluate real-time duplex colour Doppler examination with colour and pulse Doppler ultrasonography (US) (colour Doppler imaging (CDI)) for detection of the retinal detachment from membranous structures in the posterior segment. Materials and methods In 33 consecutive patients with ophthalmoscopically invisible eyes (34 eyes), CDI was performed to detect the presence or absence of retinal detachment. The diagnostic criterion for retinal detachment was whether or not spectral waveforms were detected in membranous structures with colour and pulse Doppler US. In all cases, the absence or presence of retinal detachment was confirmed by surgery.
\end{abstract}

Results In 27 of 34 eyes, membranes and/or opacities were observed in the vitreous cavity with CDI. In 12 of these 27 eyes, blood flow in those structures was detected by CDI. In all of these 12 eyes, retinal detachment was confirmed at surgery, and in $\mathbf{1 4}$ of the 15 eyes in which blood flow was not detected by CDI, absence of retinal detachment was confirmed at surgery. When a diagnosis confirmed by surgery was used as the definitive finding, CDI had a sensitivity of $92.3 \%$, specificity of $100 \%$, positive predictive value of $100 \%$, negative predictive value of $93.3 \%$, and an accuracy in the detection of retinal detachment from membranes and/or opacities in the vitreous cavity of $96.3 \%$.

Conclusion Real-time duplex colour Doppler examination with colour and pulse Doppler

US (CDI) is a noninvasive method useful for the detection of retinal detachment from vitreous opacity and/or membrane in the posterior segment.

Eye (2007) 21, 1375-1378; doi:10.1038/sj.eye.6702442; published online 9 June 2006
Keywords: regional blood flow;

ultrasonography; colour Doppler imaging; retinal detachment

\section{Introduction}

In 1991, Wong et al ${ }^{1}$ used colour Doppler ultrasonography (US) to differentiate retinal detachment from vitreous membrane, reporting $100 \%$ sensitivity in the detection of colour signals in seven cases of retinal detachment. However, Han et $a l^{2}$ reported $57 \%$ sensitivity in the detection of colour signals by only colour Doppler US in eight cases of retinal detachment, and $93 \%$ sensitivity by colour Doppler US with injection of contrast-enhancing agents.

However, although a highly accurate diagnosis is achieved using contrast-enhancing agents, the procedure involved is invasive.

Colour Doppler imaging (CDI) is real-time duplex colour Doppler examination with colour and pulse Doppler US on a B-mode background. This modality gives the approximate flow velocity of the principal vessels in the eye and orbit, enabling the sample volume to be accurately positioned on the colour Doppler display for pulse Doppler examination. ${ }^{3,4}$

CDI has been proposed as a tool for diagnosis or study of vascular disorders in the eye and orbit. Several CDI studies have examined the ocular vessels, primarily via assessment of measured velocity or calculation of resistive index. However, the velocity wave gained by CDI may be utilized not only to investigate changes in blood flow but also to detect the presence or absence of regional blood flow.

The present prospective study assessed the clinical usefulness of using colour Doppler imaging to detect the presence of arterial blood flow in the membranous structures and thereby diagnose retinal detachment in patients with opaque media.
Department of

Ophthalmology,

Mie University School of

Medicine, Tsu, Mie, Japan

Correspondence: M Ido,

Department of

Ophthalmology,

Mie University

School of Medicine,

2-174 Edobashi, Tsu,

Mie 514-8507, Japan

Tel: + 81592321111 ;

Fax: + 81592313036 .

E-mail: mss@amigo2.ne.jp

Received: 15 January 2006 Accepted in revised form:

21 April 2006

Published online:

9 June 2006 


\section{Materials and methods}

Thirty-three patients (15 male subjects, aged 15-70 years, 18 female subjects, aged 16-82 years) with invisible eyes by ophthalmoscope (34 eyes) were referred for US between October 1999 and March 2002. Ultrasonography was performed by one examiner using a LOGIQ 500 system (GE YOKOGAWA MEDICAL SYSTEMS, Tokyo, Japan) equipped with a 7.5-MHz linear array transducer.

Patients were asked to lie in the supine position with eyes closed and to gaze straight ahead while restricting ocular movement. The transducer was applied to the closed eyelids using sterile ophthalmic methylcellulose as a coupling gel and US was performed while maintaining the gel space between the eyelid and probe on the display to prevent direct contact. The greyscale mode was used at the gain from 46 to $98 \mathrm{~dB}$, and horizontal, vertical, and oblique scans through the eye were performed to detect the membranes and/or opacities in the vitreous cavity. Real-time duplex US Doppler examinations with colour and pulse Doppler (CDI) were performed at the following settings: colour and pulse Doppler gain from 14 to $16 \mathrm{~dB}$, velocity scale from 7.5 to $5.0 \mathrm{~cm} / \mathrm{s}$, wall filter at $0.4 \mathrm{~cm} / \mathrm{s}$, mechanical index within 0.41 , and thermal index within 0.53 . First, the blood flow velocity and spectral waveforms in the central retinal artery were identified and recorded with a sample volume length of $2 \mathrm{~mm}$ (Figure 1). Next, we attempted to detect the blood flow in the membranes and/or opacities using CDI at the same settings used for the central retinal artery, but at a sample volume length of $1 \mathrm{~mm}$ owing to the small size of the retinal artery. The diagnostic criterion for retinal detachment was whether or not continual waveforms with the same rates as in the central retinal artery were observed in the membranous structures.

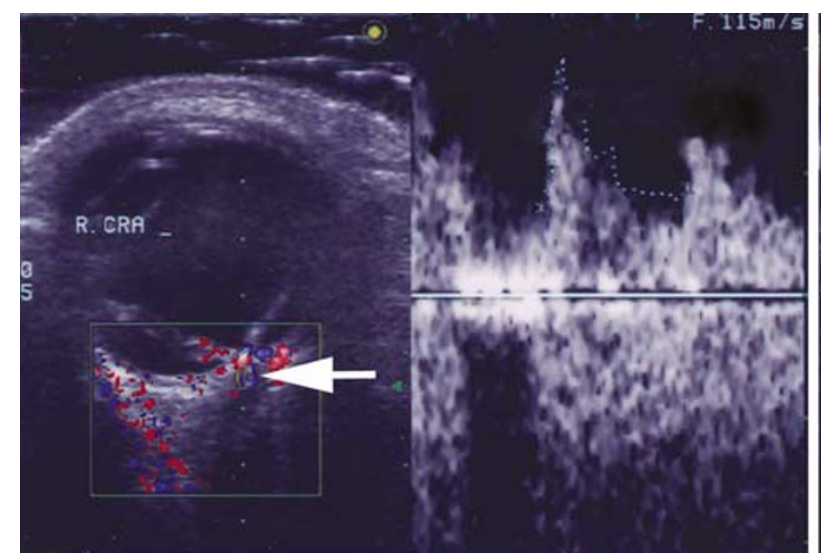

The sonographic findings were then compared with the results of surgery in all patients.

\section{Result}

In seven of 34 eyes, no membranes and/or opacities in the vitreous cavity were observed, and thus absence of retinal detachment was diagnosed without the need for CDI. In the 27 eyes in which membranes and/or opacities were observed, blood flow velocity and waveforms in the central retinal artery were recorded, and in 12 of these 27 eyes, waveforms synchronized with those of the central retinal artery were observed within parts of the membranes and/or opacities with CDI (Figure 2). In all of these 12 eyes, retinal detachment was

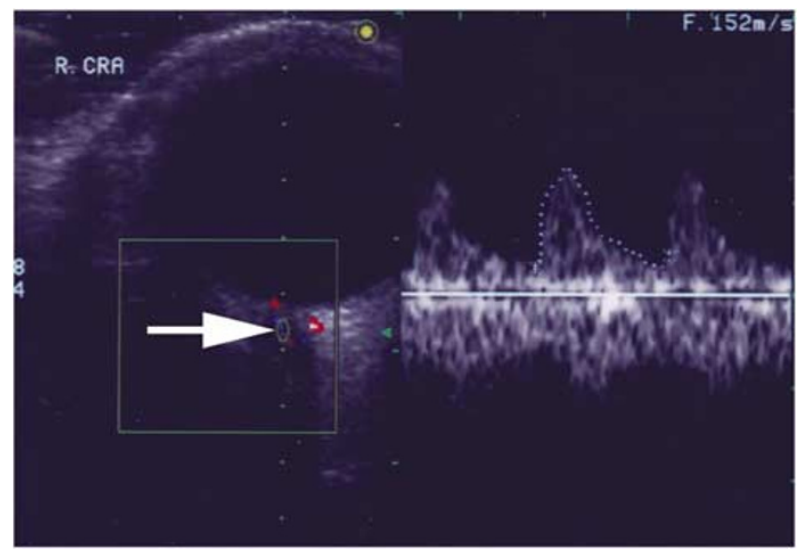

Figure 1 Duplex colour Doppler examination (CDI) of the central retinal artery in a normal volunteer. Colour visualization with colour Doppler US on a B-mode background is shown in the left display, and velocity profile (spectral waveform) with pulsed Doppler is shown in the right display (arrow: sampling position).

Figure 2 A 19-year-old male with atopic dermatitis accompanied by mature cataract and retinal detachment. A spectral waveform of the central retinal artery was obtained (left). At the same time, the waveforms obtained in the membranous structure were synchronized with those in the CRA (right). Arrow: sampling position.

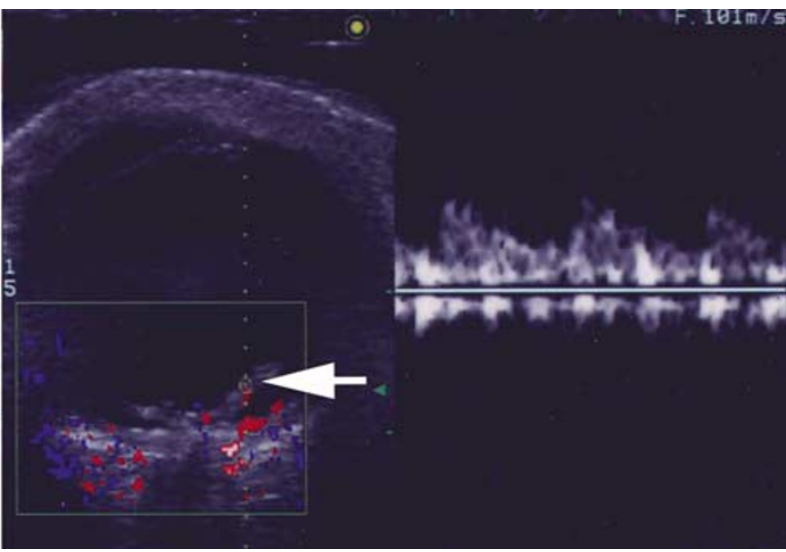


Table 1 Diagnosis by CDI and surgery

\begin{tabular}{lccc}
\hline CDI diagnosis & \multicolumn{2}{c}{ Confirmed diagnosis } & \\
\cline { 2 - 3 } & $R D(+)$ & $R D(-)$ & \\
\hline RD sign (+) & 12 & 0 & 12 \\
RD sign (-) & 1 & 14 & 15 \\
& 13 & 14 & 27 \\
\hline
\end{tabular}

Abbreviations: CDI, colour Doppler imaging; RD, retinal detachment; RD $\operatorname{sign}(+)$, presence of blood flow in the membranous structures; RD sign $(-)$, absence of blood flow in the membranous structures.

identified at surgery. In 14 of the 15 eyes in which blood flow was not detected by CDI, the absence of retinal detachment was identified at surgery. In only one eye in which blood flow was not detected by CDI, was retinal detachment identified at surgery.

Compared to the surgical results, the sensitivity of CDI was $92.3 \%$ (confidence interval (CI): 63.4-99.8\%), specificity was $100 \%$ (CI: $76.2-100 \%$ ), positive predictive value was $100 \%$ (CI: $73.1-100 \%$ ), negative predictive value was $93.3 \%$ (CI: $67.1-99.8 \%$ ), and the accuracy in differentiating retinal detachment from membranes and/or opacities in the vitreous cavity was $96.3 \%$ (CI: 80.7-99.9\%) (Table 1).

\section{Discussion}

Grey-scale US for diagnosis of retinal detachment from membranous structures in the posterior segment has obtained acceptance, and application in the evaluation of the eye with opaque media, and the requisite criteria are well established as follows: the detached retina is seen in grey-scale US as a curvilinear structure in the vitreous cavity, attached to the optic disc posteriorly and ora serrata anteriorly, with moderately strong echogenicity and relatively even thickness. Movement of the retinal detachment is restricted to movement of the eye.

The posterior hyaloid membranes or central vitreous gels are not usually attached to the optic disc or ora serrata and are visualized as structures with weaker echogenicity and more variable thickness, with greater mobility than retinal detachment. ${ }^{5,6}$

However, differentiating retinal detachment from those structures in the posterior segment is difficult in cases where the retinal detachment is shallow or localized, those structures are attached to the optic disc or ora serrata in a chronic state with high echogenicity, or those are accompanied by retinal detachment. ${ }^{7-10}$

In cases with such atypical findings in grey-scale US, colour Doppler US can play an additional, more reliable role in diagnosis, by enabling detection of blood flow in the detached retina.

The retina is supplied by the central retinal artery, which is a branch of the ophthalmic artery. After entering the optic nerve about $1 \mathrm{~cm}$ behind the eye, the central retinal artery appears at the centre of the optic disc and divides into an upper and lower branch. Each of these arteries then divides into a nasal and temporal branch known as the superior and inferior nasal arterioles and the superior and inferior temporal arterioles. These arterioles then develop smaller branches along the retinal surface. The larger vessels run in the layer of nerve fibres, and are not anastomotic with each other. ${ }^{1,2}$ Therefore, the key to diagnosis of retinal detachment is whether or not blood flow is identified in the membranous structures.

Wong et $a l^{1}$ reported the usefulness of colour flow Doppler imaging for differentiation of detached retina and membranous structures in the posterior segment. In that study, colour flow Doppler imaging identified retinal detachment in all eyes with a colour signal in the membranous structures (100\% sensitivity).

However, because colour Doppler US depends on flow velocity, vessel size, the insonation angle of a sound beam, the depth of the lesion, scanner sensitivity, and operator control, use of colour Doppler US to demonstrate flow in a vascular structure is limited. Han et $a l^{2}$ reported detection of a colour signal in $57 \%$ $(8 / 14)$ of retinal detachment cases, with a diagnostic accuracy of $81 \%$ using the colour Doppler method only. They also detected a colour signal in 93\% (13/14) of retinal detachment cases, with a diagnostic accuracy of $97 \%$ (32/33), using the colour Doppler method with intravenous injection of contrast-enhancing agents.

Real-time duplex colour Doppler examination with colour and pulsed Doppler US (CDI) provides a display of blood flow characteristics on a grey-scale B-mode background. This permits the noninvasive assessment of blood flow velocity in small blood vessels. This method has been used in ophthalmology to investigate ocular circulation with the measurement of blood flow velocity and assessment of blood flow resistance. In the present study, CDI was utilized to obtain the spectral waveform of blood flow in detached retinas, and the aim was not to measure velocity but to detect the presence or absence of blood flow in the membranous structures, thereby avoiding artefacts on the colour Doppler mode display. Thereafter, the spectral waveforms of the central retinal arteries were compared with those obtained from the membranous structures.

In our prospective study, the spectral waveform was detected in $92.3 \%(12 / 13)$ of retinal detachment cases, with a diagnostic accuracy of $96.3 \%$ (26/27). Thus, this method is as accurate as contrast-enhanced colour Doppler US, with the advantage of being noninvasive.

Only in one eye in the present study in which the spectral waveform was not detected, was retinal 
detachment identified at surgery. In that case, the vitreous opacity or membrane corresponding to the retinal detachment was not detected near the wall of the eye before surgery. The membranous structure that was observed with US was identified as the posterior hyaloid membrane during surgery, whereas very shallow retinal detachment was identified at the same time. Therefore, we believe that the detachment may have manifested or developed during surgery.

A limitation of this technique is that there are some areas on the retina where the arterial blood flow cannot be found within the precision of CDI. Therefore, when the retinal detachment is localized in the peripheral area or there are little detectable arterioles in the area of retinal detachment, it may be difficult to obtain the spectral waveforms with CDI. Moreover, diabetic retinopathy or other proliferative vascular disorders accompanied with tractional detachment were not enrolled in this study. It may be impossible to detect the blood flow of the neovascularization in the proliferative membranes, which is fed at venous pressure, because venous blood flow does not show the pulsatile waveforms with CDI such as arterial blood flow. However, it may be possible to diagnose the retinal detachment due to traction of the proliferative membranes with this technique, which utilizes the detection of arterial blood flow.

In conclusion, real-time duplex colour Doppler examination with colour and pulsed Doppler US, or CDI, is a noninvasive method that enables detection of blood flow in the membranous structures, while avoiding artefacts. This modality is useful for differentiating retinal detachment from vitreous opacity and/or membrane.

\section{References}

1 Wong AD, Cooperberg PL, Ross WH, Araki DN. Differentiation of detached retina and vitreous membrane with color flow Doppler. Radiology 1991; 178: 429-431.

2 Han SS, Chang SK, Yoon JH, Lee YJ. The use of contrastenhanced color Doppler ultrasound in the differentiation of retinal detachment from vitreous membrane. Korean J Radiol 2001; 2: 197-203.

3 Lieb WE, Cohen SM, Merton DA, Shields JA, Mitchell DG, Goldberg BB. Color Doppler imaging of the eye and orbit. Arch Ophthalmol 1991; 109: 527-531.

4 Giovagnorio F, Quaranta L, Bucci MG. Color Doppler assessment of normal ocular blood flow. J Ultrasound Med 1993; 12: 473-477.

5 Kaiser HJ, Schotzau A, Flammer J. Blood-flow velocities in the extraocular vessels in normal volunteers. Am J Opthalmol 1996; 122: 364-370.

6 Rankin SJ, Drance SM, Buckley AR, Walman BE. Visual field correlations with color Doppler studies in open angle glaucoma. J Glaucoma 1996; 5: 15-21.

7 Santos L, Capeans C, Gonzalez F, Lorenzo J, Codesido J, Salorio MS. Ocular blood flow velocity reduction after buckling surgery. Graefes Arch Clin Exp Ophthalmol 1994; 232: 666-669.

8 Stefanczyk L, Orawiec B, Gralek M, Majka R, Niwald A. Usefulness of color Doppler ultrasonography in diagnosis of retinal detachment. Klin Oczna 1996; 98: 287-290.

9 Coleman DJ, Jack RL. B-scan ultrasonography in diagnosis and management of retinal detachment. Arch Ophthalmol 1973; 90: 29-34.

10 Kerman BM, Coleman DJ. B-scan ultrasonography of retinal detachment. Ann Ophthalmol 1978; 10: 903-911. 